\title{
Web Service Recommendation Using QOS Parameters and Users Location
}

\author{
Asst.Prof. N.K. Patil ${ }^{1}$, Priyanka pawar ${ }^{2}$, Shankar More ${ }^{3}$, Bhausaheb Tupe ${ }^{4}$ \\ Computer Engg. Department, SIT,Lonavala, SIT,Lonavala ${ }^{1,2,3,4}$
}

\begin{abstract}
A Web service is a method of communication between two electronic devices over a network. Web services have been widely employed for building service-oriented applications. Recommendation techniques are very important in the fields of E-commerce and other Web-based services. One of the main difficulties is dynamically providing highquality recommendation on sparse data. In this paper, a novel collaborative filtering-based Web service recommendation algorithm is proposed, in which information contained in both ratings and profile contents are utilized by exploring latent relations between ratings, a set of dynamic features are designed to describe user preferences in multiple phases, and finally a recommendation is made by adaptively weighting the features.
\end{abstract}

Keywords: Web service, Recommendation, Quality of service (QoS), Location, Clustering, Collaborative filtering.

\section{INTRODUCTION}

WEB services are software components designed to support interoperable machine-to-machine interaction over a network, usually the Internet. Web service employs WSDL (Web Service Description Language) for interface description and SOAP (Simple Object Access Protocol) for exchanging structured information. Web services have been widely employed by both enterprises and individual developers for building service oriented applications. When developing service-oriented applications, developers first design the business process according to requirements, and then try to find and reuse existing services to build the process. Collaborative filtering is valuable in e-commerce, and for direct recommendations for music, movies, news etc. Collaborative filtering aims at learning predictive models of user preferences, interests or behavior from community data, i.e. a database of available user preferences. we describe a new model-based algorithm designed for this task, which is based on a generalization of probabilistic latent semantic analysis to continuous-valued response variables.

\section{LITERATURE SURVEY}

When developing service-oriented applications, developers first design the business process according to requirements, and then try to find and reuse existing services to build the process. Currently, many developers search services through public sites like Google Developers (developers.google.com), Yahoo! Pipes (pipes.yahoo. com), programmable Web (programmableweb.com), etc. However, none of them provide location-based QoS information for users. Such information is quite important for software deployment especially when trade compliance is concerned. Some Web services are only available in EU, thus software employing these services cannot be shipped to other countries. Without knowledge of these things, deployment of service-oriented software can be at great risk.

1. Web service QoS performance has less correlation to the locations of users.

2. No Web Service Recommendation

3. Service-oriented software can be at great risk

Copyright to IJARCCE

\section{PROPOSED SYSTEM}

This paper investigates personalized QoS value prediction for service users by employing the available past user experiences of Web services from different users. Our approach requires no additional Web service invocations.

Based on the predicted QoS values of Web services, personalized QoS-aware Web service recommendations can be produced to help users select the optimal service among the functionally equivalent ones[3].

we find that the user-observed Web service QoS performance has strong correlation to the locations of users. To enhance the prediction accuracy, we propose a location-aware Web service recommender system which employs both Web service QoS values and user locations for making personalized QoS prediction.

1. Reduce risk and deliver high-quality business processes

2. Web service QoS performance has Strong correlation to the locations of users.

\section{$3 . \quad$ User Collaboration}

4. Location information is also considered when clustering users and services.

5. Service region and User region

IV. SYSTEM ARCHITECTURE

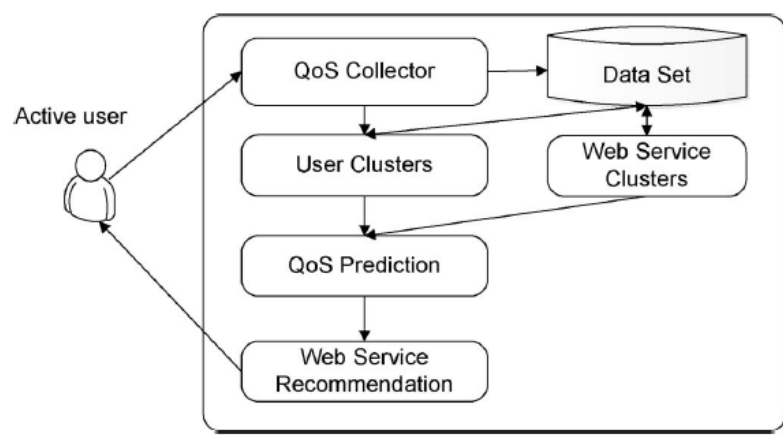

Fig1. proposed system architecture 


\section{PROPOSED ALGORITHM}

\section{Collaborative Filtering}

Collaborative Filtering (CF) is widely employed in commercial recommender systems, such as Netflix and Amazon. Com The basic idea of $\mathrm{CF}$ is to predict and recommend potential favorite items for a particular user employing rating data collected from other users[4].CF is based on processing the user-item matrix. It divide the $\mathrm{CF}$ algorithms into two broad classes:

\section{Memory based Algorithm}

Generally the task in the CF is to predict the words of particular user from a database of user votes from a sample or population of other user. We distinguish between the various $\mathrm{CF}$ algorithm in terms of the details of the "weight" calculation. There are other possible characterizations for memory based $\mathrm{CF}$.

\section{Model based algorithm}

From a probabilistic perspective, the CF task can be viewed as calculating the expected value of vote, given what we know about the user. For the active user, we wish to predict votes on as-yet unobserved items.

\section{Pearson Correlation}

Pearson Correlation Coefficient (PCC) is widely used to measure user similarity in recommender systems measures the similarity between two service users a and $u$ based on the QoS values of Web services.

The PCC similarity of two service users, Sim $(a, u)$ ranges from -1 to 1 . Positive PCC value indicates that two users have similar Web service usage experiences, while negative PCC value means that the Web service usage experiences are opposite. $\operatorname{Sim}(\mathrm{a}, \mathrm{u})=$ null when two users have no commonly invoked Web service.

\section{Top-k Algorithm}

Top-K algorithms rank the neighbors based on their PCC similarities and select the top $\mathrm{k}$ most similar neighbors for making missing value prediction. In practice, some entries in the user-item matrix have limited similar neighbors or even do not have any neighbors. Traditional Top-K algorithms ignore this problem and still include dissimilar neighbors to predict the missing value, which will greatly reduce the prediction accuracy. To attack this problem, we propose an enhanced Top-K algorithm, where neighbors with PCC similarities smaller or equal to 0 will be excluded [1].

\section{Clustering Algorithm}

The clustering method has two parts: initialization and aggregation. In the initialization part, we select non sensitive user regions for aggregation, and compute the similarity between each region pair with . To aggregate regions,
1. Select the most similar region pair (region I,region j) merge the regions to region $\mathrm{i}$ if their similarity exceeds the similarity threshold -u, otherwise stop this region aggregation process. To merge the two regions

a. Compute the sensitivity and region center of this newly merged region regioni. Remove this region from aggregation process if it becomes a sensitive one.

b. Remove similarities between regionj and other existing regions.

c. Update similarities between regioni and other existing regions.

2. Repeat the above step.

\section{CONCLUSION}

This paper presents a QoS-aware Web service recommendation approach. The basic idea is to predict Web service QoS values and recommend the best one for active users based on historical Web service QoS records. We combine prediction results generated from service regions and user regions, which achieves better results than existing approaches. We also find that the combination result is much better than the result from any single method, either the prediction generated from user regions or the one generated from Web service regions. This is because these two methods analyze the problem from different aspects and the combination of them counteracts the error of individual methods.

\section{ACKNOWLEDGEMENT}

Our thanks to the experts who have contributed towards development of the proposed system

[1] M. Alrifai and T. Risse, "Combining Global Optimization with Local Selection for Efficient QoS-Aware Service Composition,' in Proc. 18th Int'1 Conf. WWW, 2009, pp. 881-890.

[2] J. Canny, "Collaborative Filtering with Privacy via Factor Analysis," in Proc. 25th Int'l ACM SIGIR Conf., 2002, pp. 238-245.

[3] X. Chen, Z. Zheng, X. Liu, Z. Huang, and H. Sun, "PersonalizedmQoS-Aware Web Service Recommendation and Visualization," IEEE Trans. Serv. Comput., vol. 6, no. 1, pp. 3547R1st Quart., 2013.

[4] T. Hofmann, "Collaborative Filtering via Gaussian Probabilistic Latent Semantic Analysis,' in Proc. 26th Int'l ACM SIGIR Conf. Res. Dev. Inf. Retrieval, 2003, pp. 259-266.

\section{REFERENCES}

